\title{
SOLUTION OF AMBARTSUMIAN DELAY DIFFERENTIAL EQUATION IN THE $q$-CALCULUS
}

\author{
ABDULAZIZ M. ALANAZI, ABDELHALIM EBAID* \\ Department of Mathematics, Faculty of Sciences, University of Tabuk, P.O. Box 741, Tabuk 71491, Saudi \\ Arabia \\ *Corresponding author: aebaid@ut.edu.sa

\begin{abstract}
The Ambartsumian equation in view of the $q$-calculus is investigated in this paper. This equation is of practical interest in the theory of surface brightness in the Milky Way. Two approaches are applied to obtain the closed form solution. The first approach implements a direct series assumption while the second approach is based on the Adomian decomposition method. The two approaches lead to a unique power series of arbitrary powers. Furthermore, the convergence of the obtained series is theoretically proven. In addition, we showed that the present solution reduces to the results in the relevant literature when the quantum calculus parameter tends to 1 .
\end{abstract}

\section{INTRODUCTION}

In regular calculus, we usually use the limits to calculate the derivatives of any given real functions. While the quantum calculus ( $q$-calculus) provides the derivatives without implementing limits. Euler obtained the basic formulae in $q$-calculus in the eighteenth century. However, the notion of the definite $q$-derivative and $q$-integral were introduced by Jackson [1] to the first time. In the present time, there is a great interest in the applications of the $q$-calculus in various fields such as mathematics, number theory, and combinatorics [2]. Besides, Ernst [3, 4] pointed out that the majority of scientists who use $q$-calculus are physicists. In addition, Baxter [5] introduced the exact solutions of several models in Statistical Mechanics. Also, Bettaibi

Received October $8^{\text {th }}, 2020$; accepted November $3^{\text {rd }}, 2020$; published November $24^{\text {th }}, 2020$.

2010 Mathematics Subject Classification. 34A08.

Key words and phrases. $q$-series; $q$-calculus; $q$-differential equation; Ambartsumian equation.

(C)2021 Authors retain the copyrights of their papers, and all open access articles are distributed under the terms of the Creative Commons Attribution License. 
and Mezlini [6] obtained the solutions of some $q$-heat/ $q$-wave equations. In the literature [7-12], several interesting results for the $q$-calculus have been discussed by several authors.

This paper considers a $q$-calculus model of the Ambartsumian delay equation (ADE) in the form:

$$
\frac{d_{q} y}{d_{q} t}=-y(t)+\frac{1}{\rho} y\left(\frac{t}{\rho}\right), \quad q \in(0,1]
$$

such that

$$
y(0)=\lambda,
$$

where $\rho>1$ and $\lambda$ is a constant. The system (1-2) is a generalized form of the standard ADE which describes the surface brightness in the Milky Way [13]. When $q \rightarrow 1$, the system (1-2) was investigated by the authors [14-15]. However, the fractional model was solved in [16] using the homotopy transform analysis method by means of the Caputo's definition. Here, we consider the $q$-derivative to deal with the present model. In the literature, there are several analytical methods to deal with the system (1-2) such as the Adomian decomposition method (ADM) [17-18], the homotopy perturbation method (HPM) [19-20], and the homotopy analysis method (HAM) [16].

In this paper, two different approaches are suggested to analytically solve the system (1-2). The first approach is a regular power series approach while the second is based on the ADM. Such approaches are preferred here, especially, in proving the convergence of the resulting series solution. The paper is organized as follows. The main aspects of the $q$-calculus are presented in Section 2. In addition, a basic lemma for the integrals arise from the ADM is to be proved in Section 2. Sections 3 discusses the application of the $q$-calculus to solving the current model. Section 4 is devoted to the application of the ADM. In addition it will be shown that the present solution reduces to that one in the literature as $q \rightarrow 1$. Section 5 includes an analysis of convergence. Finally, section 6 outlines the conclusions.

\section{Preliminaries}

Let $q \in \mathbb{R}$ and $n \in \mathbb{N}$, then $[n]_{q}$ is defined as (first chapter in [21])

$$
[n]_{q}=\frac{1-q^{n}}{1-q}
$$

and as $q \rightarrow 1$, we have

$$
\lim _{q \rightarrow 1}[n]_{q}=n .
$$

The $q$-factorial $[n]_{q}$ ! of a positive integer $n$ is given by

$$
[n]_{q} !=[1]_{q} \times[2]_{q} \times[3]_{q} \times \cdots \times[n]_{q},
$$


and as $q \rightarrow 1$, we have

$$
\begin{aligned}
\lim _{q \rightarrow 1}[n]_{q} ! & =[1]_{1} \times[2]_{1} \times[3]_{1} \times \cdots \times \lim _{q \rightarrow 1}[n]_{q} \\
& =1 \times 2 \times 3 \times \cdots \times n=n !
\end{aligned}
$$

The definition of $q$-differential is $d_{q} f(t)=f(t)-f(q t)$ and the $q$-derivative of a function $f(t)$ is defined by $[21]$

$$
D_{q} f(t):=\frac{d_{q} f(t)}{d_{q} t}=\frac{f(t)-f(q t)}{(1-q) t}, \quad t \neq 0
$$

such that

$$
\lim _{q \rightarrow 1} D_{q} f(t)=f^{\prime}(t)
$$

if $f$ is differentiable at $t$, and we have at $t=0$ that

$$
D_{q} f(0)=\lim _{t \rightarrow 0} D_{q} f(t)
$$

According to (2.5) we have

$$
D_{q} t^{n}=[n]_{q} t^{n-1} .
$$

The definite Jackson $q$-integral is defined by

$$
\int_{0}^{t} f(\tau) d_{q} \tau=(1-q) t \sum_{j=0}^{\infty} q^{j} f\left(q^{j} t\right)
$$

and hence,

$$
\int_{0}^{t} D_{q} f(\tau) d_{q} \tau=f(t)-f(0)
$$

In order to apply the ADM on the system (1.1-1.2), we need to introduce and prove the following lemma.

2.1. Lemma 1. For $q \in(0,1]$, we have

$$
\int_{0}^{t} \tau^{n} d_{q} \tau=\frac{t^{n+1}}{[n+1]_{q}} .
$$

\section{Proof:}


From the definite Jackson $q$-integral given by Eq. (2.9), we obtain

$$
\begin{aligned}
\int_{0}^{t} \tau^{n} d_{q} \tau & =(1-q) t \sum_{j=0}^{\infty} q^{j}\left(q^{j} t\right)^{n} \\
& =(1-q) t^{n+1} \sum_{j=0}^{\infty}\left(q^{n+1}\right)^{j} \\
& =(1-q) t^{n+1}\left(\frac{1}{1-q^{n+1}}\right) \\
& =\frac{t^{n+1}}{[n+1]_{q}} .
\end{aligned}
$$

\section{Direct Series SOlution}

In order to solve Eq. (1.1), we assume the solution in the series form:

$$
y(t)=\sum_{n=0}^{\infty} a_{n} t^{n}
$$

and therefore

$$
\begin{aligned}
\frac{d_{q} y}{d_{q} t} & =\sum_{n=0}^{\infty}[n]_{q} a_{n} t^{n-1}, \\
& =\sum_{n=1}^{\infty}[n]_{q} a_{n} t^{n-1}, \quad \text { where } \quad[0]_{q}=0 \\
& =\sum_{n=0}^{\infty}[n+1]_{q} a_{n+1} t^{n} .
\end{aligned}
$$

Substituting (3.1) and (3.2) into (1.1), yields

$$
\begin{aligned}
\sum_{n=0}^{\infty}[n+1]_{q} a_{n+1} t^{n} & =-\sum_{n=0}^{\infty} a_{n} t^{n}+\frac{1}{\rho} \sum_{n=0}^{\infty} a_{n}\left(\frac{t}{\rho}\right)^{n} \\
& =-\sum_{n=0}^{\infty} a_{n} t^{n}+\sum_{n=0}^{\infty}\left(\frac{1}{\rho^{n+1}}\right) a_{n} t^{n} \\
& =\sum_{n=0}^{\infty}\left(\frac{1}{\rho^{n+1}}-1\right) a_{n} t^{n}
\end{aligned}
$$

or

$$
\sum_{n=0}^{\infty}\left[[n+1]_{q} a_{n+1}-\left(\frac{1}{\rho^{n+1}}-1\right) a_{n}\right] t^{n}=0
$$

which requires that

$$
[n+1]_{q} a_{n+1}-\left(\frac{1}{\rho^{n+1}}-1\right) a_{n}=0
$$


Therefore

$$
a_{n+1}=\left(\frac{\rho^{-(n+1)}-1}{[n+1]_{q}}\right) a_{n}, \quad n \geq 0
$$

From (3.6), we have

$$
\begin{aligned}
& a_{1}=\left(\frac{\rho^{-1}-1}{[1]_{q}}\right) a_{0}, \\
& a_{2}=\left(\frac{\rho^{-2}-1}{[2]_{q}}\right) a_{1}=\left(\frac{\left(\rho^{-1}-1\right)\left(\rho^{-2}-1\right)}{[1]_{q} \times[2]_{q}}\right) a_{0}, \\
& a_{3}=\left(\frac{\rho^{-3}-1}{[3]_{q}}\right) a_{2}=\left(\frac{\left(\rho^{-1}-1\right)\left(\rho^{-2}-1\right)\left(\rho^{-3}-1\right)}{[1]_{q} \times[2]_{q} \times[3]_{q}}\right) a_{0}, \\
& \cdot \\
& a_{n}=\left(\frac{\left(\rho^{-1}-1\right)\left(\rho^{-2}-1\right)\left(\rho^{-3}-1\right) \ldots\left(\rho^{-n}-1\right)}{[1]_{q} \times[2]_{q} \times[3]_{q} \times \cdots \times[n]_{q}}\right) a_{0} .
\end{aligned}
$$

This $n$-term coefficient can expressed in terms of the $q$-factorial $[n]_{q}$ ! as

$$
a_{n}=\frac{a_{0}}{[n]_{q} !} \prod_{i=1}^{n}\left(\rho^{-i}-1\right), \quad n \geq 1 .
$$

Thus

$$
\begin{aligned}
y(t) \quad & =a_{0}+\sum_{n=1}^{\infty} a_{n} t^{n}, \\
& =a_{0}+a_{0} \sum_{n=1}^{\infty} \frac{t^{n}}{[n]_{q} !} \prod_{i=1}^{n}\left(\rho^{-i}-1\right), \\
& =a_{0}\left[1+\sum_{n=1}^{\infty} \frac{t^{n}}{[n]_{q} !} \prod_{i=1}^{n}\left(\rho^{-i}-1\right)\right] .
\end{aligned}
$$

Applying the initial condition (1.2) on (3.9), yields $a_{0}=\lambda$. Hence, the closed-form solution of the system $(1.1-1.2)$ is finally given by

$$
y(t)=\lambda\left[1+\sum_{n=1}^{\infty} \frac{t^{n}}{[n]_{q} !} \prod_{i=1}^{n}\left(\rho^{-i}-1\right)\right],
$$

which is the solution of ADE in the $q$-calculus. Moreover, the solution (3.10) as $q \rightarrow 1$ reduces to

$$
\begin{aligned}
y(t) & =\lambda\left[1+\lim _{q \rightarrow 1} \sum_{n=1}^{\infty} \frac{t^{n}}{[n]_{q} !} \prod_{i=1}^{n}\left(\rho^{-i}-1\right)\right], \\
& =\lambda\left[1+\sum_{n=1}^{\infty} \frac{t^{n}}{\left(\lim _{q \rightarrow 1}[n]_{q} !\right)} \prod_{i=1}^{n}\left(\rho^{-i}-1\right)\right], \\
& =\lambda\left[1+\sum_{n=1}^{\infty} \frac{t^{n}}{n !} \prod_{i=1}^{n}\left(\rho^{-i}-1\right)\right],
\end{aligned}
$$

which is the same closed form solution obtained by the authors [14] for the standard model of Ambartsumian equation. 


\section{Application of the ADM}

Integrating Eq. (1.1) and based on Eq. (2.10), we have

$$
y(t)=\lambda+\int_{0}^{t}\left(\frac{1}{\rho} y\left(\frac{\tau}{\rho}\right)-y(\tau)\right) d_{q} \tau
$$

Following the ADM [17-18], we assume that

$$
y(t)=\sum_{k=0}^{\infty} y_{k}(t)
$$

and hence,

$$
\begin{aligned}
& y_{0}(t)=\lambda \\
& y_{k+1}(t)=\int_{0}^{t}\left(\frac{1}{\rho} y_{k}\left(\frac{\tau}{\rho}\right)-y_{k}(\tau)\right) d_{q} \tau, \quad k \geq 1 .
\end{aligned}
$$

At $k=1$, Eq. (4.3) gives

$$
\begin{aligned}
y_{1}(t) & =\int_{0}^{t}\left(\frac{1}{\rho} y_{0}\left(\frac{\tau}{\rho}\right)-y_{0}(\tau)\right) d_{q} \tau \\
& =\lambda\left(\rho^{-1}-1\right) \int_{0}^{t} d_{q} \tau \\
& =\lambda\left(\rho^{-1}-1\right) \frac{t}{[1]_{q}}
\end{aligned}
$$

where Lemma 1 is implemented to calculate the involved integral. Similarly, at $k=2$, we have

$$
\begin{aligned}
y_{2}(t) & =\int_{0}^{t}\left(\frac{1}{\rho} y_{1}\left(\frac{\tau}{\rho}\right)-y_{1}(\tau)\right) d_{q} \tau, \\
& =\lambda\left(\rho^{-1}-1\right)\left(\rho^{-2}-1\right) \int_{0}^{t} \frac{\tau}{[1]_{q}} d_{q} \tau, \\
& =\lambda\left(\rho^{-1}-1\right)\left(\rho^{-2}-1\right) \frac{t^{2}}{[1]_{q}[2]_{q}} .
\end{aligned}
$$

Proceeding as above, we obtain

$$
y_{k}(t)=\lambda\left(\rho^{-1}-1\right)\left(\rho^{-2}-1\right)\left(\rho^{-3}-1\right) \ldots\left(\rho^{-k}-1\right) \frac{t^{k}}{[1]_{q}[2]_{q}[3]_{q} \ldots[k]_{q}} .
$$

From Eq. (2.3), we can rewrite Eq. (4.6) as

$$
y_{k}(t)=\lambda \prod_{j=1}^{k}\left(\rho^{-j}-1\right)\left(\frac{t^{k}}{[k]_{q} !}\right), \quad k \geq 1 .
$$


Thus

$$
\begin{aligned}
y(t) \quad & =y_{0}(t)+\sum_{k=1}^{\infty} y_{k}(t), \\
& =\lambda+\lambda \sum_{k=1}^{\infty} \frac{t^{k}}{[k]_{q} !} \prod_{j=1}^{k}\left(\rho^{-j}-1\right), \\
& =\lambda\left[1+\sum_{k=1}^{\infty} \frac{t^{k}}{[k]_{q} !} \prod_{j=1}^{k}\left(\rho^{-j}-1\right)\right],
\end{aligned}
$$

which is the same closed form series solution that was obtained in the previous section. The series (3.11) which is equivalent to (4.8) will be proved for convergence in the next section.

\section{Analysis of COnVergence}

In order to proving the convergence of (3.11), we assume that

$$
a_{n}=\frac{1}{[n]_{q} !} \prod_{i=1}^{n}\left(\rho^{-i}-1\right), \quad n \geq 1
$$

Accordingly, we obtain the theorem below.

\section{Theorem 1:}

The radius of convergence of the series $(3.11)$ is $\left(\frac{1}{1-q}\right) \forall q \in(0,1]$.

\section{Proof:}

Assume that $\mu$ is the radius of convergence. Therefore, we have from (5.1) and the ratio test that

$$
\begin{aligned}
\frac{1}{\mu} & =\lim _{n \rightarrow \infty}\left|\frac{a_{n+1}}{a_{n}}\right|, \\
& =\lim _{n \rightarrow \infty}\left|\frac{\prod_{i=1}^{n+1}\left(\rho^{-i}-1\right)}{[n+1]_{q} !} \times \frac{[n]_{q} !}{\prod_{i=1}^{n}\left(\rho^{-i}-1\right)}\right| .
\end{aligned}
$$

From (2.3), we observe that

$$
[n+1]_{q} !=[1]_{q} \times[2]_{q} \times[3]_{q} \times \cdots \times[n]_{q} \times[n+1]_{q}=[n]_{q} ![n+1]_{q} .
$$

Inserting (5.3) into (5.2), we obtain

$$
\frac{1}{\mu}=\lim _{n \rightarrow \infty}\left|\frac{\left(\rho^{-(n+1)}-1\right) \prod_{i=1}^{n}\left(\rho^{-i}-1\right)}{[n]_{q} ![n+1]_{q}} \times \frac{[n]_{q} !}{\prod_{i=1}^{n}\left(\rho^{-i}-1\right)}\right| .
$$


Simplifying (5.4), it then follows

$$
\begin{aligned}
\frac{1}{\mu} & =\lim _{n \rightarrow \infty}\left|\frac{\rho^{-(n+1)}-1}{[n+1]_{q}}\right|, \\
& =\left|\lim _{n \rightarrow \infty}\left(\rho^{-(n+1)}-1\right) / \lim _{n \rightarrow \infty}\left([n+1]_{q}\right)\right|, \\
& =\left|-1 /\left(\frac{1}{1-q}\right)\right|, \quad \text { where } \rho>1, \\
& =|1-q|, \\
& =1-q \quad \forall q \in(0,1]
\end{aligned}
$$

which completes the proof, where the following property:

$$
\lim _{n \rightarrow \infty}[n+1]_{q}=\lim _{n \rightarrow \infty}\left(\frac{1-q^{n+1}}{1-q}\right)=\frac{1}{1-q} \quad \forall q \in(0,1],
$$

was implemented in deducing (5.5). It is noticed from (5.6) that as $q \rightarrow 1$ then $\frac{1}{\mu} \rightarrow 0$. Hence, the series (3.11) has an infinite radius of convergence at such special case which is in full agreement with the results obtained by [14] for the standard ADE.

\section{Conclusion}

In this paper, the quantum calculus was applied to generalize the Ambartsumian equation. The resulting $q$-differential equation was analytically solved via the power series approach and the ADM. The convergence of the obtained power series was theoretically proven. The implemented approaches led to the same closed form series solution. In addition, we showed that the present solution reduces to the results in the literature when the quantum calculus parameter tends to 1 . Finally, the present work can be further extended to explore several physical models in view of the $q$-calculus.

Availability of Data and Material: Not applicable.

Authors' Contributions: All authors read and approved the final manuscript and all authors have agreed to the authorship and the order of authorship for this manuscript.

Conflicts of Interest: The author(s) declare that there are no conflicts of interest regarding the publication of this paper.

\section{REFERENCES}

[1] F.H. Jackson, On a $q$-Definite Integrals, Quart. J. Pure Appl. Math. 41 (1910), 193-203.

[2] G.E. Andrews, $q$-Series: Their Development and Applications in Analysis, Number Theory, Combinatorics, Physics, and Computer Algebra, CBMS series, vol. 66, Amer. Math. Soc., Providence, 1986. 
[3] T. Ernst, The history of $q$-calculus and a new method (LicentiateThesis), U.U.D.M. Report 2000: 16; http://www.math.uu.se/thomas/Lics.pdf.

[4] T. Ernst, A method for q-Calculus, J. Nonlinear Math. Phys. 10(4) (2003), 487-525.

[5] R. Baxter, Exact Solved Models in Statistical Mechanics, Academic Press, New York, 1982.

[6] N. Bettaibi, K. Mezlini, On the use Of the $q$-Mellin transform to solve some $q$-heat and $q$-wave equations, Int. J. Math. Arch. 3(2) (2012), 446-455.

[7] Y. Li, Z.-M. Sheng, A deformation of quantum mechanics, J. Phys. A: Math. Gen. 25 (1992), $6779 ? 6788$.

[8] M.H. Annaby, Z.S. Mansour, $q$-Fractional Calculus and Equations, Springer, Heidelberg, New York, 2012.

[9] I. Koca, E. Demirci, On local asymptotic stability of $q$-fractional nonlinear dynamical systems, Appl. Appl. Math. 11 (2016), 174-183.

[10] N.A. Rangaig, C.T. Pada, V.C. Convicto, On the existence of the solution for $q$-Caputo fractional boundary value problem, Appl. Math. Phys. 5 (2017), 99-102.

[11] Y. Tang, T. Zhang, A remark on the $q$-fractional order differential equations, Appl. Math. Comput. 350 (2019), $198-208$.

[12] L. Chanchlani, S. Alha, J. Gupta, Generalization of Taylor's formula and differential transform method for composite fractional $q$-derivative, Ramanujan J. 48 (2019), 21-32.

[13] V.A. Ambartsumian, On the fluctuation of the brightness of the milky way, Doklady Akad Nauk USSR, 44 (1994), $223-226$.

[14] J. Patade, S. Bhalekar, On Analytical Solution of Ambartsumian Equation, Natl. Acad. Sci. Lett. 40 (2017), $291-293$.

[15] T. Kato, J.B. McLeod, The functional-differential equation $y^{\prime}(x)=a y(\lambda x)+b y(x)$, Bull. Amer. Math. Soc. 77 (1971), 891-935.

[16] D. Kumar, J. Singh, D. Baleanu, S. Rathore, Analysis of a fractional model of the Ambartsumian equation, Eur. Phys. J. Plus, 133 (2018), 259.

[17] A. Alshaery, A. Ebaid, Accurate analytical periodic solution of the elliptical Kepler equation using the Adomian decomposition method, Acta Astron. 140 (2017), 27-33.

[18] H.O. Bakodah and A. Ebaid, The Adomian decomposition method for the slip flow and heat transfer of nanofluids over a stretching/shrinking sheet, Rom. Rep. Phys. 70 (2018), 115.

[19] A. Patra, S. Saha Ray, Homotopy perturbation sumudu transform method for solving convective radial fins with temperature-dependent thermal conductivity of fractional order energy balance equation, Int. J. Heat Mass Transfer. 76 (2014), 162-170.

[20] Z. Ayati, J. Biazar, On the convergence of Homotopy perturbation method, J. Egypt. Math. Soc. 23 (2015), 424-428.

[21] V.G. Kac, P. Cheung, Quantum Calculus, Springer-Verlag, New York, 2002. 